\title{
Corrección de mielomeningocele intrautero: Reporte del primer caso en Ecuador
}

\section{Case report: First intrauterine myelomeningocele correction in Ecuador}

\author{
Pablo Santillán Roldán ${ }^{1, *}$, Andrés Cepeda Mora1', Fernanda Herrera Zambrano', Lorena Guacales Zambrano, \\ Pablo Armas Cruz ${ }^{1}$, Natalie Arroyo ${ }^{1}$ \\ 1 Clínica Atlas. Quito, Ecuador.
}

Fecha de recepción: 17 de marzo de 2021 / Fecha de aprobación: 25 de abril de 2021

\begin{abstract}
This is the case of a 26 year old patient, at week 27 of gestational age and with a prenatal diagnosis of myelomeningocele. It was decided to enter the clinic to perform the first correction of the spina bifida defect in Ecuador, with the support of a multidisciplinary team of national and foreign professionals. This procedure was carried out within international parameters and standards for the management of this pathology, with the equipment, supplies and trained personnel to carry this surgery to a successful outcome, both for the fetus and its mother. It is demonstrated that our country has the capacity of both human resources and equipment to solve highly complex pathologies.
\end{abstract}

Key words: Spina bifida, myelomeningocele, fetal surgery, intrauterine surgery.

\section{RESUMEN}

Se trata del caso de una paciente de 26 años, en la semana 27 de edad gestacional y con un diagnóstico prenatal de mielomeningocele. Se decide ingresar a la clínica para realizar la primera corrección de defecto de espina bífida del Ecuador, con apoyo de un equipo multidisciplinario de profesionales nacionales y extranjeros. Este procedimiento se llevó a cabo dentro de parámetros y estándares internacionales de manejo de esta patología, contando con los equipos, insumos y el personal capacitado para llevar esta cirugía a un desenlace exitoso, tanto para el feto como su madre. Queda demostrado que en nuestro país se cuenta con la capacidad tanto de recursos humanos y equipos para resolver patologías de gran complejidad.

Palabras clave: Espina bífida, mielomeningocele, cirugía fetal, cirugía intrauterina.

\section{Introducción}

racias a los avances en el diagnóstico por imagen las detecciones de los pacientes con problemas del tubo neural son cada vez más frecuentes, estos defectos se presentan de 33-48 por cada 100.000 nacidos vivos a nivel mundial.

La cirugía fetal representa una oportunidad de tratamiento intra-uterino única para malformaciones congénitas que de otra manera conllevarían morbi-mortalidad importante. A raíz del estudio MOMS (manejo del mielomeningocele), el mielomeningocele se convirtió en la indicación mas frecuente para la realización de cirugía fetal[1].

La terapia fetal intraútero data de los años 1963 con la primera transfusión intraperitoneal exitosa de sangre a un feto con eritroblastosis fetal por Sir William Liley, a raíz de lo cual se ha ido desarrollando a pasos acelerados por lo que actualmente se podría clasificar de acuerdo al diagnóstico de la patología y edad gestacional en tres tipos de procedimientos: cirugía mínimamente invasiva y cirugía abierta que se los realiza desde la 
mitad del segundo trimestre hasta la mitad del tercer trimestre (18-26 semanas) y terapia intraparto extra uterina o procedimientos EXIT (ex útero intrapartum therapy)[1].

El mielomeningocele consiste en el defecto en el cierre del tubo neural hasta la $6^{\text {ta }}$ semana de gestación, con una mortalidad entre 10\%-30\% hasta los primeros 5 años de vida. Los pacientes con diagnóstico de mielomeningocele presentan un sin número de complicaciones neurológicas entre las más comunes esta la hidrocefalia en el $80 \%$ de los casos, así como paraplejia y malformacion de Chiary tipo II, incontinencia, disfunción sexual, alteraciones mentales y deformidades esqueléticas[2].

\section{Reporte del caso}

Se trata de una paciente femenina de 26 años de edad, de 27 semanas de gestación, sin antecedentes patológicos personales, ni alérgicos, con 3 antecedentes quirúrgicos ninguno relacionado con la patología actual. Acudió a control a las 20 semanas donde por medio de ecografía se diagnóstica al producto con un defecto del cierre del tubo neural L4-S1 (mielomeningocele) (Figura 1).

Al examen físico los signos vitales dentro de parámetros normales, abdomen gestante, tamaño que se correlaciona a edad gestacional, suave, depresible, feto único, frecuencia cardiaca: 140 lpm, movimientos presentes. Exámenes de laboratorio maternos dentro de rangos adecuados para su edad y sexo.

Se coordinó un equipo multidisciplinario de profesionales nacionales y extranjeros conformados por ginecólogos expertos en cirugía intraútero, neurocirujano, anestesiólogos, neonatólogos instrumentistas y personal a fin, además de contar con los equipos adecuados para monitorización en tiempo real de los parámetros maternos para una adecuada evolución durante la cirugía.

Es así que el pasado 28 de enero de 2021 en la ciudad de Quito, en la Clínica Atlas se llevó a cabo la primera cirugía intraútero para corrección de un mielomeningocele. Misma que se desarrolló en varias fases.

Dentro del manejo anestésico. En el preoperatorio se administró a la paciente profilaxis antibiótica con cefalosporina de $2^{a}$ generación, cefazolina $2 \mathrm{~g}$, además profilaxis para aspiración pulmonar, ranitidina 50 mg IV y metoclopramida $10 \mathrm{mg}$ IV 30 minutos previo a la cirugía, adicional precalentamiento con manta de aire forzado.

Se realizó monitorización continua básica no invasiva, frecuencia cardiaca, frecuencia respiratoria, electrocardiograma con cable de 5 puntas, oximetría de pulso, temperatura, capnografía y monitor de relajación neuromuscular (monitor General Electric) e invasiva con la colocación de catéter arterial radial derecho $\mathrm{N}^{\circ} 20$ para monitorización de la tensión arterial, muestreo de gases arteriales, se realizó técnica neuroaxial epidural a nivel de L3 - L4 con un catéter peridural para analgesia posoperatoria con bomba elastomérica a $5 \mathrm{ml} / \mathrm{h}$ de bupivacaina $0,125 \%$.

En el inicio de la anestesia general se colocaron equipos de protección en zonas de presión además medias para tromboprofilaxis mecánica, realizamos preoxigenación con mascarilla facial a $8 \mathrm{ltm}$ y se aplicó inducción de secuencia rápida con propofol 2,5 mg/kg, lidocaína $2 \mathrm{mg} / \mathrm{kg}$ y rocuronio 0,9 mg/kg previa infusión de remifentanilo 0,25 mcg/ $\mathrm{kg} / \mathrm{min}$, la intubación

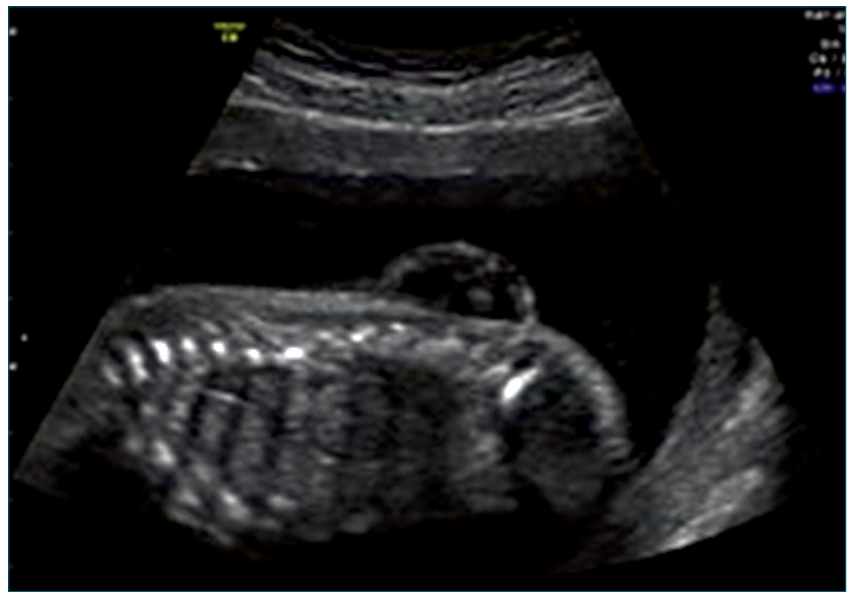

Figura 1. Vista longitudinal de la columna vertebral y espina bífida abierta. Defecto del tubo neural a nivel L4-S1. Mielomeningocele.

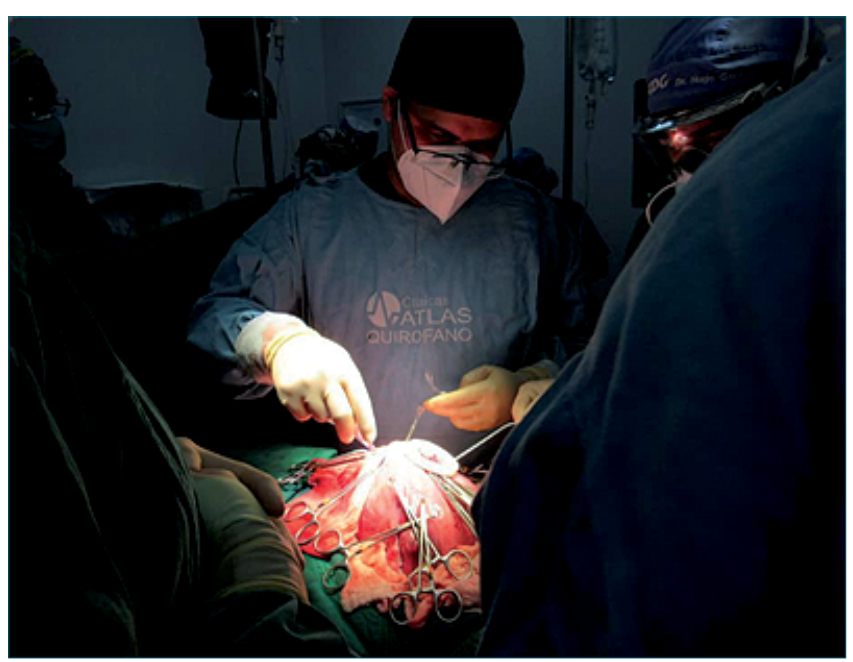

Figura 2.

oral se realizó con videolaringoscopio (Medcaptain pala N³), tubo endotraqueal 7.0 al primer intento, posterior a lo cual se acopla a la ventilación mecánica con parámetros protectivos (volumen tidal $6 \mathrm{ml} / \mathrm{kg}$, frecuencia respiratoria 14-16 por minuto, PEEP 6) con una capnometria de $32 \mathrm{mmHg}$, además con co-carga con cristaloides balanceados a $3 \mathrm{ml} / \mathrm{kg}$ más infusión continua de noradrenalina para mantener tensión arterial media sobre los $65 \mathrm{mmHg}$ y bolos de noradrenalina de $6-8 \mathrm{mcg}$ intravenosos o efedrina 12 mg intravenosos en caso de hipotensión.

La fluidoterapia se realizó con cristaloides balanceados (lactato de Ringer) a 80 ml/h, y evaluando hidratación por objetivos con monitor no invasivo de variables dinámicas biorreactancia (CHEETA) y ecografía transtoracica en puntos de valoración pulmonar y cardiaca.

Para el mantenimiento se utilizó remifentanilo $0,25 \mathrm{mcg} / \mathrm{kg} /$ min, sevofluorano de 1,5 a 2 CAM, asociado con una infusión de nitroglicerina de $0,25-0,5 \mathrm{mcg} / \mathrm{kg} / \mathrm{min}$, sulfato de magnesio con una dosis de carga de 4-6 $\mathrm{g}$ en 30 minutos y dosis de 
mantenimiento de 1-2 g/h para obtener una inhibición uterina adecuada.

La preparación de fármacos anestésicos para el feto se lo realizó mediante técnica estéril envasando fentanilo $20 \mathrm{mcg} / \mathrm{kg}$, rocuronio $0,8 \mathrm{mg} / \mathrm{kg}$ y atropina $20 \mathrm{mcg} / \mathrm{kg}$ por vía intramuscular; se preparó adrenalina: 20 mcg/kg por separado en caso de bradicardia fetal.

Durante todo este manejo el equipo de ginecólogos, cirujanos materno-fetales y neurocirujanos pediatrico en el lapso de 1 h 40 minutos, realizo la reparación del defecto del cierre de tubo neural a nivel L4- S1 (mielomeningocele) (Figura 3) mismo que se llevó a cabo con éxito. Feto fue monitorizado mediante ecografía Doppler de arteria umbilical durante todo el procedimiento de manera intermitente. Una vez finalizada la cirugía se procede con educción anestésica sin complicaciones manteniendo tocolisis a base de sulfato de magnesio a $1 \mathrm{~g} / \mathrm{h}$ y soporte vasoactivo durante las primeras $4 \mathrm{~h}$, paciente fue transportada a cuidados intermedios para monitorización hemodinámica continua y monitoreo fetal continuo.

Paciente es dada de alta de nuestra institución 96 h después de la intervención quirúrgica hemodinámicamente estable con monitoreo fetal clasificación I y régimen tocolítico vía oral para el domicilio.

\section{Discusión}

El mielomeningocele es la patología que con mayor frecuencia se corrige mediante cirugía abierta, ya que presenta un mejor pronóstico relacionado a la corrección posnatal del mismo. La cirugía fetal abierta se realiza mediante histerotomía y posterior exteriorización del feto por lo que la complejidad del procedimiento y la técnica anestésica va a propiciar mayores cambios hemodinámicos con requerimientos de una tocolisis más agresiva, si se la compara con los procedimientos mínimamente invasivos[2]-[4].

La intervención de un equipo multidisciplinario es fundamental para optimizar el manejo del binomio materno fetal debido al riesgo y complicaciones que pueden desencadenarse[4].

El tratamiento anestésico está enfocado en el bienestar hemodinámico de la madre y el feto además de garantizar las condiciones adecuadas para la realización del procedimiento por ende es importante considerar los agentes que van a ser empleados y sus repercusiones en la circulación feto-placentaria así como el paso de los fármacos hacia el feto. La técnica anestésica recomendada es la combinación de anestesia general más catéter peridural para manejo de analgesia postoperatorio[5],[6].

Los objetivos anestésicos deben incluir la inmovilidad fetal, adecuada relajación uterina y mantener la hemodinamia materna y fetal[2],[7].

La valoración preoperatoria debe ser clara y con conocimiento total de la historia clínica materna además se debe realizar estudios de imagen que permitan evaluar la condición y presentación fetal así como posibles anomalías uteroplacentarias, es pertinente alistar hemoderivados ante la posibilidad de complicaciones hemorrágicas propias del procedimiento[2]. Las directrices internacionales recomiendan mantener el esquema de ayuno preoperatorio de $2 \mathrm{~h}$ para líquidos claros y $6 \mathrm{~h}$ para alimentos sólidos además de la profilaxis antibiótica, contra

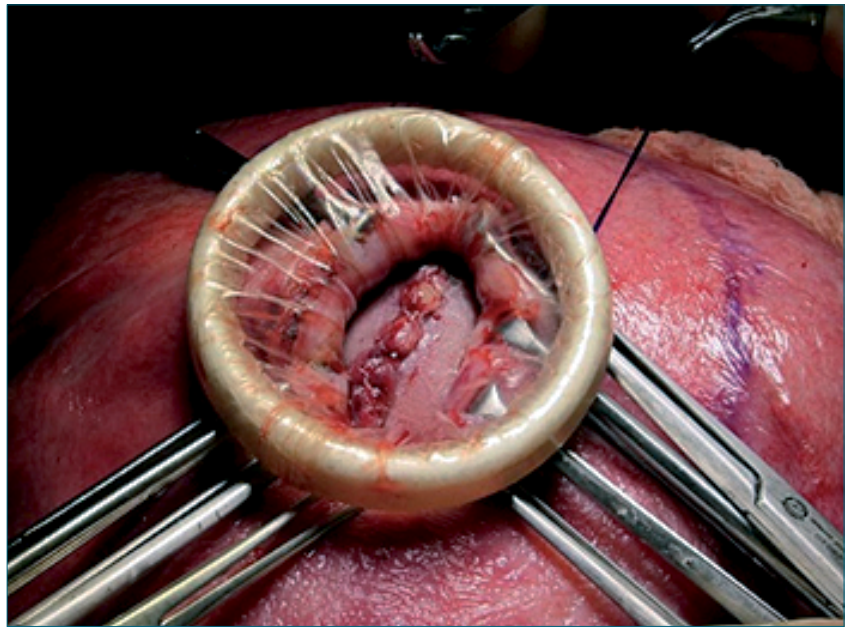

Figura 3. Histerotoma y reparo de defecto del mielomeningocele.

broncoaspiración y trombo profilaxis mecánica y prevención de la hipotermia mediante pre-calentamiento 30 minutos previo a ingresar a sala[1].

Es imperativo considerar los cambios fisiológicos que se convierten en un abordaje conocido de las vías respiratorias difíciles, como una condición de estómago lleno, menor capacidad residual funcional y edema de la mucosa de la vía aérea[6].

Se debe realizar monitorización continua básica, presión arterial no invasiva, pulsioxiemtría, electrocardiograma, capnografía, temperatura, diuresis además el requerimiento de una línea arterial para control estricto de la presión arterial y la toma de muestras seriadas para registro de gases arteriales, analizador de gases anestésicos por la necesidad de altas dosis de agentes inhalados para la relajación uterina, y un catéter venoso central, para usar agentes vasoactivos[4].

Previo al inicio de anestesia general se coloca catéter peridural para manejo de analgesia posoperatoria. La anestesia general se realiza con inducción de secuencia rápida, así fármacos como el propofol a dosis de $2 \mathrm{mg} / \mathrm{kg}$, fentanil a dosis de 1 a $2 \mu \mathrm{g} / \mathrm{kg}$ o remifentanil a dosis de $0,5 \mu \mathrm{g} / \mathrm{kg}$. El relajante muscular sigue siendo la succinilcolina a $\mathrm{mg} / \mathrm{kg}$ para así realizar la intubación en los primeros 45-60 segundos[3].

Seguido del mantenimiento balanceado con anestésicos inhalatorios y opioides intravenosos a dosis elevadas que sumado a los agentes tocoliticos generan un gran compromiso hemodinámico por lo que se requiere la infusión de vasoactivos como fenilefrina o noradrenalina para mantener una adecuada circulación utero-placentaria y la perfusión a los demás órganos de la madre[1],[2],[8].

La ventilación mecánica se realiza con parámetros protectivos para así optimizar la oxigenación de la madre y del producto, garantizando además una adecuada ventilación mediante la capnografía apropiada para paciente gestante con valores entre 30-34 mmHg[1].

La relajación uterina es un paso crítico en este tipo de procedimientos para propiciar las condiciones ideales para la exposición fetal, por lo que el uso de halogenados a dosis elevadas (al menos 2 CAM) para obtener relajación uterina profunda, sin embargo, se ha demostrado que a 2,4 CAM o mayores re- 
duce el flujo umbilical durante el procedimiento quirúrgico, así como estudios que reportan efectos adversos a nivel de sistema nervioso central y cardiaco haciendo especial énfasis en la disfunción ventricular y valvular en el feto y la madre; por lo que se puede administrar infusión de nitroglicerina desde 0,5 a 1 $\mathrm{mcg} / \mathrm{kg} / \mathrm{min}$ con bolos de rescate entre 50-200 mcg intravenosos y así lograr disminuir el requerimiento de los anestésicos inhalados para la relajación uterina. A partir de la histerorrafia se utiliza sulfato de magnesio en dosis de carga de 4 a 6 $\mathrm{g}$ intravenoso en 30 minutos posteriormente se continua con infusión a 1-2 g/h manteniéndola hasta 48 h posquirúrgicas; también es posible utilizar atosiban intravenoso, nifedipino oral o terbutalina subcutánea como regímenes posoperatorios para tocolisis[2],[3].

La fluidoterapia debe mantenerse con patrón restrictivo de $80 \mathrm{ml} / \mathrm{h}$ dado el alto riesgo de edema pulmonar de la paciente gestante sumado a la intensa tocolisis y los anestésicos volátiles que puede incrementar dicho riesgo, siendo de aproximadamente el $25 \%$ de los pacientes indistintamente sea cirugía abierta o mínimamente invasivo. Motivo por el cual la monitorización de variables dinámicas como el índice de gasto cardiaco, la variabilidad del volumen sistólico mediante métodos no invasivos como la bioreactancia o la ecografía pulmonar y cardiaca nos permitirá realizar una fluidoterapia guiada por objetivos[9].

La monitorización del feto se la puede realizar con ecografía continua o intermitente, medición de gases de arteria umbilical, pulsioximetria fetal o electrodos para oximetría cerebral sin embargo, estos últimos presentan algunas limitantes. La monitorización ecográfica Doppler es la herramienta más utilizada ya sea con la medición del flujo diastólico en los vasos umbilicales o la arteria cerebral media o el índice de pulsatilidad. Es prioritario evitar la hipotermia fetal mediante la amnioinfusión de lactato ringer calentado, además para mantener el volumen uterino adecuado y evitar la compresión del cordon umbilical y así la hipoxemia fetal[8].

Dado que existe una respuesta fisiológica por parte del feto tanto hormonal como hemodinámica a los estímulos dolorosos entre las 16 y las 24 semanas de gestación en adelante; el manejo anestésico fetal para garantizar la inmovilidad durante el procedimiento se lo realiza a base de fentanil 0,2 mg/kg, rocuronio $1-3 \mathrm{mg} / \mathrm{kg}$ y para prevenir o como tratamiento de bradicardia fetal secundaria a los anestésicos se administra atropina $0,02 \mathrm{mg} / \mathrm{kg}$ o adrenalina $1 \mathrm{mcg} / \mathrm{kg}$ como droga de rescate, en el caso de requerir transfusión de hemoderivados se lo realizará con concentrados de los glóbulos rojos leucoreducidos $\mathrm{O} \mathrm{rH}$ negativo a dosis de $5 \mathrm{ml} / \mathrm{kg}$ con un cálculo de la volemia fetal entre 120-160 ml/kg[6].

El manejo posoperatorio se lo debería realizar en una unidad de cuidados intensivos obstétricos para vigilancia estricta de los parámetros hemodinamicos maternos y fetales, así como también el régimen tocolítico empleado durante el posoperatorio monitorizando los reflejos osteotendinosos, nivel de consciencia y niveles plasmáticos en caso del sulfato de magnesio, es primordial mantener un balance hídrico negativo para evitar congestión pulmonar. Además, el esquema analgésico mediante catéter peridural con anestesico local y opioide sumado a los analgésicos intravenosos logra mantener un nivel de oxitocina así como factores de la cascada inflamatoria bajos con lo que se relaciona con una menor tasa de complicaciones materno -fetales[5],[7],[9].

Entre las complicaciones asociadas a estos procedimientos durante el transoperatorio destacan el desprendimiento de placentario con la consiguiente hemorragia y posible hipoxemia fetal por lo que se enfatiza en conseguir una relajación uterina y analgesia fetal adecuada para optimizar la exposición del producto. La separación de las membranas corioamnioticas ocurre en el $65,6 \%$ de los casos de acuerdo a la serie reportada por Licci y cols., así como la ruptura prematura de membranas en $2 \%$, oligohidramnios en $13 \%$ a en el período posoperatorio inmediato y la dehiscencia de la histerorrafia al momento de la cesarea en el $21,5 \%$ de los casos. A pesar de las precauciones tomadas durante la cirugía y los avances en cuanto a la técnica quirúrgica, existe el riesgo latente en todos estos procedimientos de una labor de parto prematura por lo que se debe preparar a las pacientes y al equipo sanitario para ese momento[9].

\section{Referencias}

1. Chestnut $D$, et al. Chestnut's Obstetric Anesthesia Principles and practice. Sixth Edit. Elsevier Health; 2019. 1324 p.

2. Maldonado C, Torres P, Riquelme D. Implications and anesthetic considerations for fetal surgery. Rev Chil Anest. 2019;48(4):32430. https://doi.org/10.25237/revchilanestv48n04.07.

3. Goonasekera CD, Skelton VA, Zebian B, Nicolaides K, Araujo Lapa D, Santorum-Perez M, et al. Peri-operative management of percutaneous fetoscopic spina-bifida repair: a descriptive review of five cases from the United Kingdom, with focus on anaesthetic implications. Vol. 43, International Journal of Obstetric Anesthesia. Churchill Livingstone; 2020. p. 97-105. https://doi. org/10.1016/j.ijoa.2020.04.005.

4. Devoto JC, Alcalde JL, Otayza F, Sepulveda W. Anesthesia for myelomeningocele surgery in fetus. Childs Nerv Syst. 2017 Jul;33(7):1169-75. https://doi.org/10.1007/s00381-017-3437-7 PMID:28547209

5. Kodali BS, Bharadwaj S. Foetal surgery: Anaesthetic implications and strategic management. Vol. 62, Indian Journal of Anaesthesia. Indian Society of Anaesthetists; 2018. p. 717-23.

6. Van de Velde M, De Buck F. Fetal and maternal analgesia/anesthesia for fetal procedures. Fetal Diagn Ther. 2012;31(4):201-9. https://doi.org/10.1159/000338146 PMID:22538233

7. Manrique S, Maiz N, García I, Pascual M, Perera R, Arévalo S, et al. Maternal anaesthesia in open and fetoscopic surgery of foetal open spinal neural tube defects: A retrospective cohort study. Eur J Anaesthesiol. 2019 Mar;36(3):175-84. https://doi.org/10.1097/ EJA.0000000000000930 PMID:30507621

8. Ramírez MV. Anesthesia for fetal surgery. Volume 40. Revista Colombiana de Anestesiologia. Sociedad Colombiana de Anestesiologia y Reanimacion SCARE; 2012. pp. 268-72.

9. Licci M, Guzman R, Soleman J. Maternal and obstetric complications in fetal surgery for prenatal myelomeningocele repair: a systematic review. Neurosurg Focus. 2019 Oct;47(4):E11. https:// doi.org/10.3171/2019.7.FOCUS19470 PMID:31574465 